\title{
Protein Expression in Vascular Endothelial Cells Obtained from Human Peripheral Arteries and Veins
}

\author{
Annemarie E. Silver ${ }^{a}$ Demetra D. Christou ${ }^{a}$ Anthony J. Donato ${ }^{a}$ \\ Stacy D. Beske ${ }^{a}$ Kerrie L. Moreau ${ }^{a, b}$ Katherine A. Magerko ${ }^{a}$ Douglas R. Seals $^{a, b}$

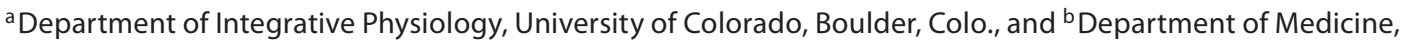 \\ University of Colorado, Denver, Colo., USA
}

\section{Key Words}

Nuclear factor- $\kappa \mathrm{B} \cdot$ Nitric oxide $\cdot$ Oxidants $\cdot$ Antioxidants

\begin{abstract}
Studying molecular mechanisms of vascular endothelial function in humans is difficult in part because of limited access to arteries. Access to peripheral veins is more practical. We determined if differences in protein expression of endothelial cells $(E C)$ collected from a peripheral artery are reflected in measurements made on EC obtained from peripheral veins. EC were collected from the brachial artery and an antecubital vein of 106 healthy adults ( 60 men and 46 women, age 18-77 years). Quantitative immunofluorescence was used to measure protein expression of endothelial nitric oxide synthase (eNOS), Ser-1177 phosphorylated eNOS, manganese superoxide dismutase, nitrotyrosine, xanthine oxidase and nuclear factor-кB p65. Protein expression in EC obtained from brachial artery and antecubital vein sampling was moderately to strongly related $(r=0.59-0.81$, all $p<$ 0.0001 , mean $r=0.70$ ). Moreover, differences between subgroups in the lowest and highest tertiles of protein expression in EC obtained from arterial samples were consistently reflected in EC obtained from venous collections. These findings indicate that interindividual and group differences in expression of several proteins involved in nitric oxide pro-
\end{abstract}

duction, oxidant production, antioxidant defense and inflammatory signaling in EC obtained from brachial artery sampling are consistently reflected in EC obtained from venous samples. Thus, EC collected from peripheral veins may provide a useful surrogate for $\mathrm{EC}$ obtained from arteries for measurements of EC protein expression in humans.

Copyright $\odot 2009$ S. Karger AG, Basel

The vascular endothelium plays a key role in vascular physiology and pathophysiology in humans $[1,2]$. Differences in the properties of the vascular endothelium including the expression of proteins associated with nitric oxide production, oxidant formation, antioxidant defense and inflammation influence vascular endothelial function and disease risk [3, 4]. As a result, it is important to establish techniques that can assess the expression of these proteins in the vascular endothelium in humans.

In 1999, Feng et al. [5] first described a novel technique of obtaining endothelial cells (EC) from intact arteries and veins of patients using guide wires advanced through a catheter. Subsequently, Colombo et al. [6] used a similar procedure of collecting EC from arteries and veins of healthy subjects and patients to establish a method of determining protein expression of endothelium-derived factors using quantitative immunofluorescence. The im-

\section{KARGER}

(C) 2009 S. Karger AG, Basel

Fax +41613061234

E-Mail karger@karger.ch

www.karger.com
Accessible online at:

www.karger.com/jvr
Dr. Douglas R. Seals

Department of Integrative Physiology

University of Colorado, UCB 354

Boulder, CO 80309 (USA)

Tel. +1 303492 5305, Fax +1 303492 6778, E-Mail seals@colorado.edu 
plications of this technique are vast because it allows proteomic assessment of EC obtained from living humans without the use of surgical procedures.

Obviously, to most directly study the molecular mechanisms underlying arterial endothelial function or disease processes, arterial tissue is required. However, because of the invasiveness of catheterizations required to obtain arterial samples and the associated risks to human subjects, assessment of protein expression in EC obtained from peripheral veins represents an attractive potential alternative. Use of cells collected from veins to evaluate EC protein expression would allow more widespread application of this promising technique, including use in healthy human subjects. Indeed, the importance of comparing EC protein expression from arteries and veins in vivo recently has been emphasized [7].

Accordingly, the purpose of this study was to gain further insight into this issue in men and women free of clinical cardiovascular disease. To do so, we first examined the relations between protein expression measured in EC collected from a peripheral artery and veins within individual subjects in the overall sample using simple linear regression analysis. We then assessed expression in EC in subgroups of subjects with arterial values in the lowest and highest tertiles for each protein to determine if venous values consistently depict group differences in arterial values.

\section{Materials and Methods}

\section{Subjects}

A total of 106 adults (60 men and 46 women) were studied. All subjects had blood pressure $<140 / 90 \mathrm{~mm} \mathrm{Hg}$, body mass index $<40 \mathrm{~kg} / \mathrm{m}^{2}$ and were free of cardiovascular disease, diabetes, and other clinical diseases as assessed by medical history, physical examination, resting electrocardiogram and blood chemistries. Men older than 40 years and women older than 50 years of age were further evaluated with electrocardiogram and blood pressure responses to incremental treadmill exercise performed to volitional exhaustion [8]. Subjects were nonsmokers and not taking medications or dietary supplements (for example, antioxidants) that could influence the results. All procedures were approved by the Human Research Committee of the University of Colorado at Boulder. The nature, benefits and risks of the study were explained to the volunteers and their written informed consent was obtained prior to participation.

\section{Study Procedures}

All measurements were performed at the University of Colorado at Boulder General Clinical Research Center after an overnight fast and a 24-hour abstention from alcohol and exercise.
Anthropometric Measurements

Body weight was measured to the nearest $0.1 \mathrm{~kg}$ with a digital scale (Itin Scale Co. Inc., Brooklyn, N.Y., USA). Body mass index was calculated as weight in kilograms divided by squared height in meters.

\section{Vascular EC Protein Expression}

The procedures used for collection of vascular EC and measurement of EC protein expression have been previously described in detail $[9,10]$. Briefly, EC were collected from the brachial artery and from an antecubital vein of the contralateral arm. For the collection, 2 sterile J-wires were briefly advanced (approx. $4 \mathrm{~cm}$ beyond the tip of the catheter) and retracted through an 18-gauge catheter. The wires were transferred to a dissociation buffer solution and immediately brought to the wet lab for washing and fixation. The cells were recovered by washing and centrifugation. Cells were fixed with 3.7\% formaldehyde and plated on poly-L-lysine-coated slides (Sigma Chemical, St. Louis, Mo., USA). The slides were stored in a $-80^{\circ} \mathrm{C}$ freezer until they were removed for staining.

For immunofluorescence staining, 2 control human umbilical vein EC (HUVEC) slides and 16 subject slides were selected for each staining batch. The HUVEC slides used for the present study were all prepared from the same batch and passage of cells. Cells were rehydrated with PBS and rendered permeable using $0.1 \%$ Triton X-100 (Alfa Aesar, Ward Hill, Mass., USA). After blocking nonspecific binding sites with 5\% donkey serum (Jackson Immunoresearch, West Grove, Pa., USA), cells were incubated with monoclonal antibodies for one of the following: endothelial nitric oxide synthase (eNOS; Transduction Laboratories, San Jose, Calif., USA), Ser-1177 phosphorylated endothelial nitric oxide synthase (P-eNOS; Calbiochem, San Diego, Calif., USA), manganese superoxide dismutase (MnSOD; Research Diagnostics, Concord, Ma., USA), nitrotyrosine (Abcam, Cambridge, Mass., USA), xanthine oxidase (United States Biological, Swampscott, Mass., USA) and nuclear factor- $\kappa$ B p 65 (NF- $\kappa$ B; Novus, Littleton, Colo., USA). Cells were next incubated with CY3-conjugated secondary antibodies (Jackson Immunoresearch or Research Diagnostics Inc., Concord, Mass., USA).

For analysis, slides were viewed using a fluorescence microscope (Eclipse 600; Nikon, Melville, N.Y., USA) and images were digitally captured by a Photometrics CoolSNAPfx digital camera (Roper Scientific Inc., Tucson, Ariz., USA). Slides were systematically scanned to identify EC, as evidenced by cell staining of von Willebrand factor, and nuclear integrity was confirmed using DAPI (4',6'-diamidino-2-phenylindole hydrochloride) staining. Once EC with intact nuclei were identified, images were captured and analyzed using Metamorph Software (Universal Imaging Corp., Downingtown, Pa., USA) to quantify the intensity of CY3 staining (that is, average pixel intensity), hence quantifying EC protein expression. In order to limit photobleaching, the technician exposed each cell to light in the wavelength range of the CY3 staining for only the brief time required to acquire an image with the digital camera. The software program allows for systematic quantification of staining intensity and eliminates human subjectivity from the measurement. Values are reported as ratios of EC protein expression/HUVEC. Reporting ratios minimizes the possible confound of differences in intensity of staining among different staining sessions. A single technician analyzed each batch of slides. Technicians were blinded to subject identity during the staining and analysis procedures. 


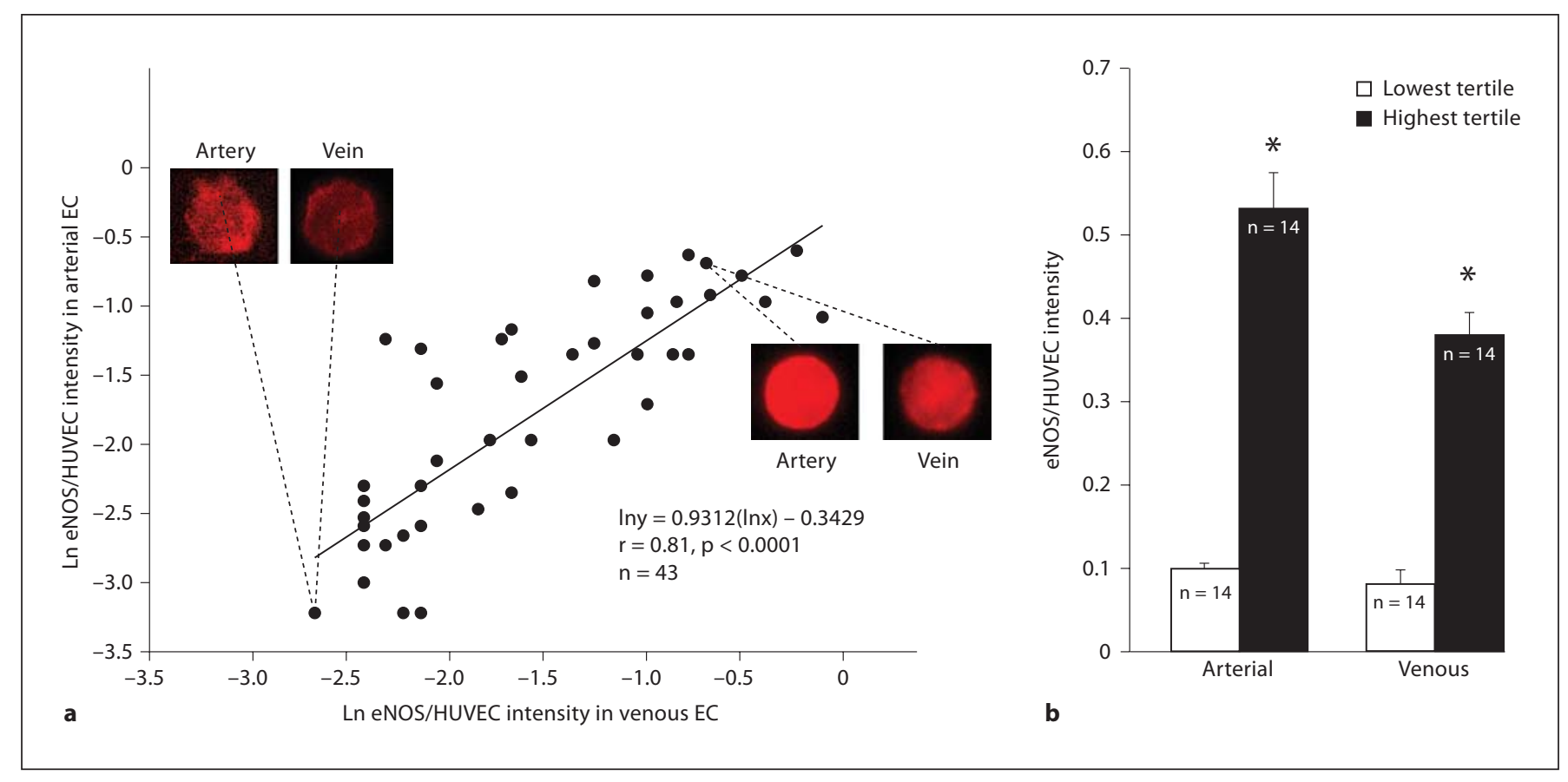

Fig. 1. a Log-transformed relation of protein expression of eNOS in EC obtained from arterial and venous samples. b Protein expression in EC acquired from arteries and veins in the lowest and highest tertiles of eNOS in arterial samples. ${ }^{*} \mathrm{p}<0.0001$, lowest vs. highest tertiles.

\section{Data Analysis}

Statistical analyses were performed with SPSS (version 11.0.3) Statistical significance for all analyses was set at $\mathrm{p}<0.05$.

To determine the relation between protein expression of EC obtained from arterial and venous samples within individual subjects, simple linear regression analysis was performed. Protein expression in EC from arterial samples was entered as the dependent variable, whereas protein expression in EC from venous samples was entered as the independent variable. The zero-order correlation coefficient derived from regression analysis was used to determine the association between arterial and venous sample EC protein expression. Residual analyses to test the validity of the regression model assumptions were performed for all models and violations were remedied using data transformation.

To determine if the protein expression of EC obtained from veins reflects group differences in protein expression of $\mathrm{EC} \mathrm{ob-}$ tained from arteries, we selected subgroups of subjects with EC protein expression from arterial samples within the lowest and highest tertiles for each protein of interest. We first confirmed that EC protein expression from arterial samples significantly differed between the 2 groups and then determined if these differences also could be depicted using protein expression of EC obtained from venous samples. Group differences were determined by $\mathrm{t}$ tests for independent sample comparisons.

\section{Results}

Protein expression of eNOS, P-eNOS, MnSOD, nitrotyrosine, xanthine oxidase and NF- $\kappa \mathrm{B}$ p65 were moderately to strongly related in EC collected from arteries and veins within individual subjects in the pooled sample cohort $(\mathrm{r}=0.59-0.81, \mathrm{p}<0.0001$; fig. 1a-6a). The mean correlation coefficient for the 6 proteins examined was $r=$ 0.70 . Significant differences between groups of subjects in the lowest versus highest tertiles of protein expression for arterial samples were consistently demonstrated using venous samples (fig. 1b-6b).

\section{Discussion}

This study represents the first systematic assessment of the relation between expression of proteins in EC obtained from arteries and veins of humans, and the first such data on healthy subjects. The key finding was that the expression of selective proteins in EC collected from peripheral veins is moderately to strongly related to that determined in EC obtained from the brachial artery. Moreover, group 

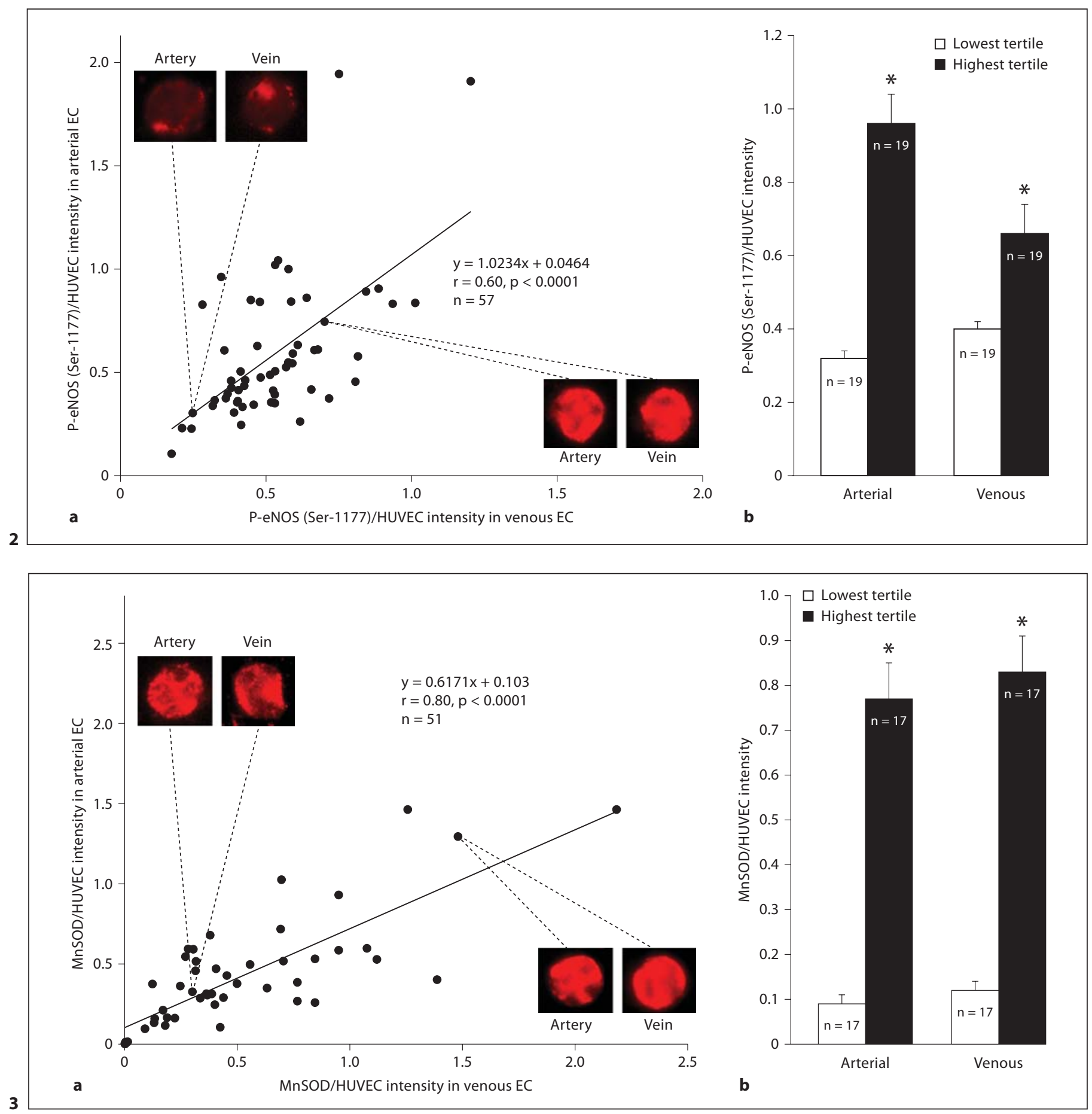

Fig. 2. a Relation of abundance of P-eNOS (Ser-1177) in EC obtained from arterial and venous samples. b Content in EC acquired from arteries and veins in the lowest and highest tertiles of P-eNOS in arterial EC samples. ${ }^{*} \mathrm{p}<0.0001$, lowest vs. highest tertiles.

Fig. 3. a Relation of protein expression of MnSOD in EC obtained from arterial and venous samples. b Protein expression in EC acquired from arteries and veins in the lowest and highest tertiles of MnSOD protein expression in arterial samples. ${ }^{*} \mathrm{p}<0.0001$, lowest vs. highest tertiles. 

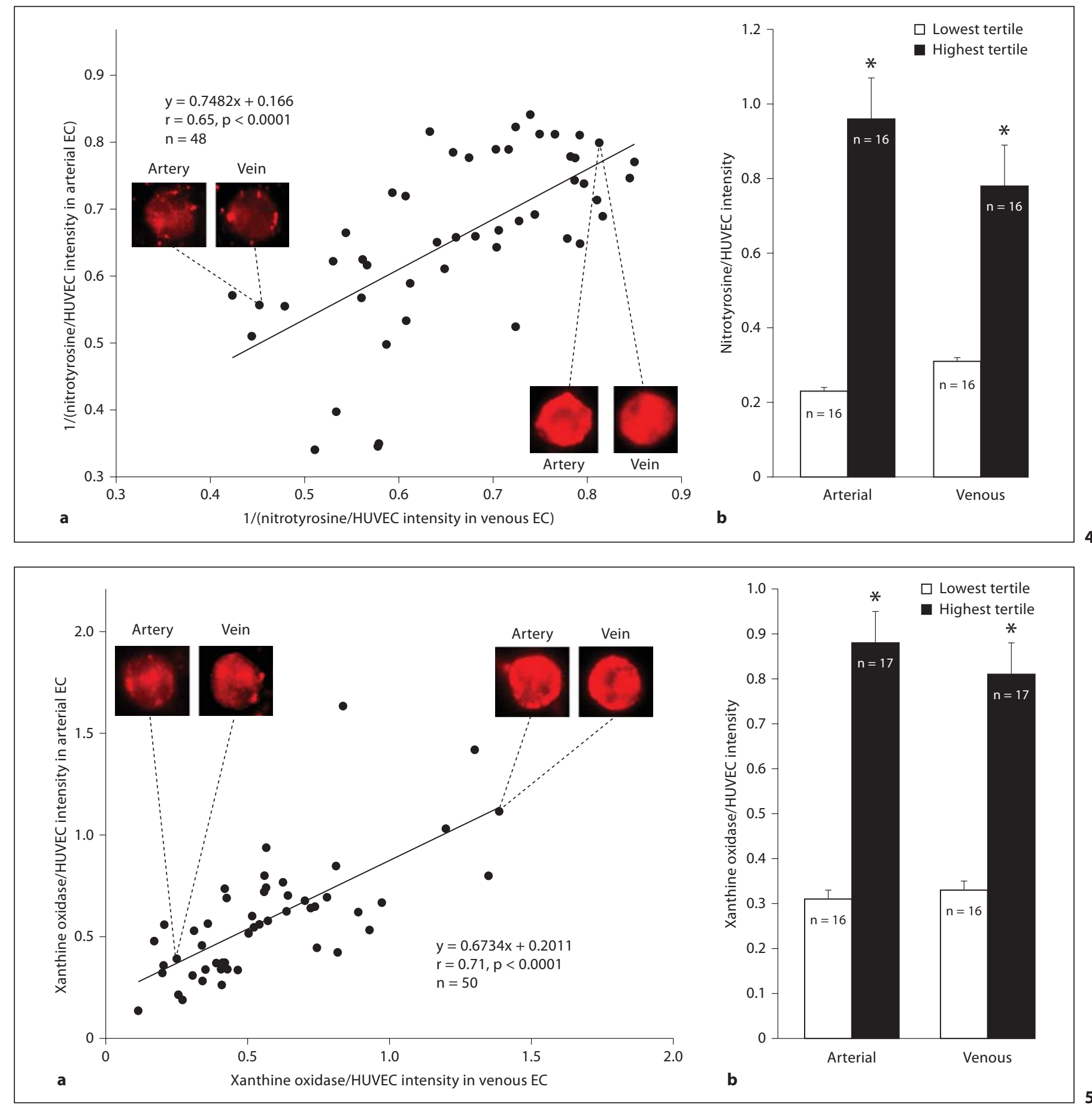

Fig. 4. a Relation of abundance of nitrotyrosine between EC obtained from arterial and venous samples. $\mathbf{b}$ Content in EC acquired from arteries and veins in the lowest and highest tertiles of nitrotyrosine in arterial samples. ${ }^{*} \mathrm{p}<0.0001$, lowest vs. highest tertiles.

Fig. 5. a Relation of protein expression of xanthine oxidase in EC obtained from arterial and venous samples. b Protein expression in EC acquired from arteries and veins in the lowest and highest tertiles of xanthine oxidase in arterial samples. ${ }^{*} \mathrm{p}<0.0001$, lowest vs. highest tertiles. 


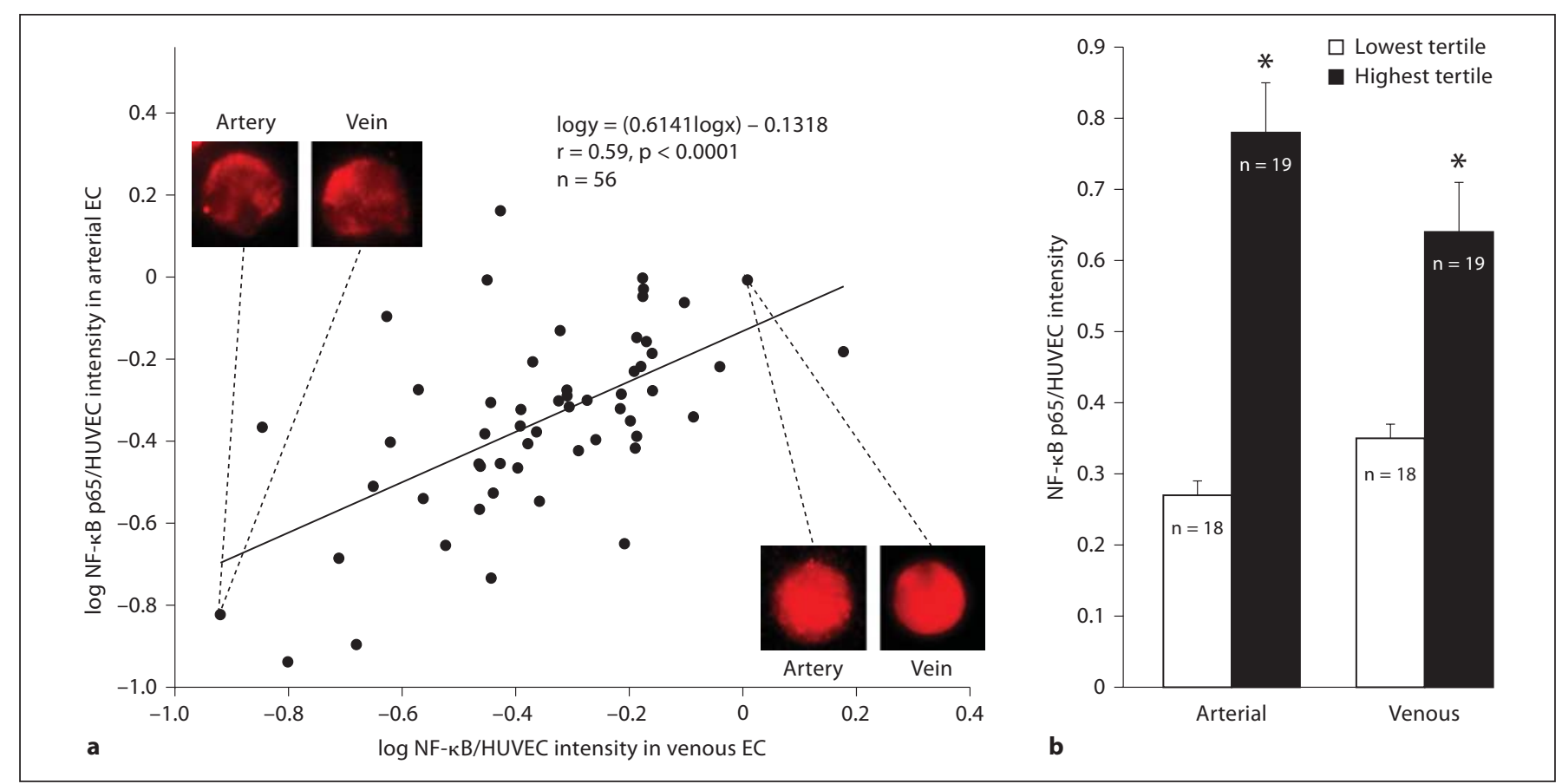

Fig. 6. a Log-transformed relation of protein expression of NF-кB p65 in EC obtained from arterial and venous samples. b Protein expression in EC acquired from arteries and veins in the lowest and highest tertiles of NFкB p65 EC in arterial samples. ${ }^{*} \mathrm{p}<0.0001$, lowest vs. highest tertiles.

differences in expression of these proteins measured from brachial artery sampling are consistently reflected in measurements obtained in EC from peripheral veins. Thus, our results provide initial support that EC collected from peripheral veins may be useful surrogates for protein expression in EC from peripheral arteries.

Limited observations in patients with cardiovascular disease and group comparisons of healthy young and older adults are consistent with the findings of the present study. In the original study establishing the present procedures, Colombo et al. [6] reported values for EC protein expression of nitrotyrosine, NF- $\mathrm{KB}$ nuclear translocation, cyclooxygenase- 2 and eNOS obtained from the radial arteries and superficial forearm veins of 5 patients with severe chronic heart failure. Based on our analysis of the individual data reported (their table 2), the correlations between arterial and venous values were $r=0.48$ 0.96 (mean $r=0.80$ ), which is similar to that observed in the present study in a larger group of healthy adults. These correlations likely underestimate, perhaps substantially, the true physiological relations between the expression of these proteins in EC obtained from arteries and veins because of the error associated with each of the respective measurements, which would act to lower the correlation coefficients. Consistent with these correlations and the subgroup analyses from the present study, our laboratory recently found significant age group differences in nitrotyrosine and NF- $\mathrm{KB}$ p 65 using EC collected from both the brachial artery and peripheral veins in healthy men [9].

Thus, taken together, our findings [present study, 9] and those of Colombo et al. [6] support the idea that expression of proteins in EC obtained from venous sampling is consistently and significantly related to expression in EC obtained from peripheral arteries in humans. One possible explanation for the associations between protein expression in EC sampled from peripheral arteries and veins is that both cell types are exposed to similar systemic factors. Indeed, numerous circulating factors, including sex hormones [11], angiotensin II [12], lipoproteins [13] and cytokines [14], modulate the expression of endothelium-derived molecules. Some genetic influences also would be common to vascular EC from arteries and veins.

We wish to emphasize that absolute expression of proteins in EC can differ in arteries and veins [15]. Although 
this was not the primary issue addressed in the present study, our data indicate that among the 6 proteins studied, absolute values were lower, similar or even higher in EC obtained from veins as opposed to the brachial artery. The previous observations of Colombo et al. [6] in patients with cardiovascular disease are in agreement with our findings. Absolute differences in protein expression between EC obtained from arteries and veins would be expected given the different structure and functions of arteries and veins as well as the hemodynamic stimuli and local factors to which they are exposed. However, despite differences in flow, structure and vascular function, expression of several proteins is significantly related in EC obtained from arterial and venous sampling. It is possible that, at least for the proteins assessed in the present and previous studies [6,9], systemic factors may exert a major influence on EC protein expression, consistent with the recent conclusions of Guzik et al. [15]. Future studies will be required to determine if the expression of other endothelial proteins is related in EC obtained from arteries and veins. The expression of some endothelial proteins may differ in EC from arteries and veins because local factors may have greater impact compared with systemic influences.

Our study has several limitations. We examined a limited number of proteins and our results cannot be generalized to other endothelium-derived factors. Only healthy adults were studied, and our findings are limited to peripheral arteries and veins. We also recognize the semiquantitative nature of immunofluorescence staining. However, the technique used in the present study has been validated against immunoblotting previously by others [5] and more recently by our laboratory [10]. Moreover, we utilized the Metamorph software analysis system to reduce error and, thus, more objectively quantify protein expression. It is also important to emphasize that other techniques for determining protein expression such as Western blotting also are semiquantitative in nature. The fact that significant relations were found in the present study despite limitations that would act to weaken or completely mask those relations argues for the strength of the physiological associations observed. Finally, it should be noted that for proteins that serve as enzymes, such as eNOS, the influence on vascular endothelial function is a consequence of activity rather than solely expression. As a result, enzyme activity could differ in arteries and veins despite similar abundance of protein.

In conclusion, the present findings indicate that the expression of selective proteins involved in the regulation of vascular endothelial function and disease risk is related in EC acquired from the brachial artery and antecubital veins. Individual and group differences in the expression of these proteins in EC collected from arteries are consistently reflected in EC obtained from venous sampling. Because collection of EC from peripheral superficial veins is quick and minimally invasive, our findings may have important implications for the use of EC obtained from venous collections for the assessment of endothelial proteins in both healthy humans and those with clinical disease. Indeed, it is possible that cells acquired from veins could be used to assess EC protein expression in large population studies and/or clinical trials using this technique.

\section{Acknowledgements}

We thank Ashok Talreja, Anita Banjaree and Thierry LeJemtel for their assistance in developing the techniques used in the present study, as well as Adam Levy, Ashley Depaulis, Brooke Lawson, Andrew Radford and Rhea Chiang for their assistance in data acquisition and analysis. This work was supported by National Institutes of Health awards AG006537, AG013038, AG022241, AG000279, AG029337, HL007851 and RR00051.

\section{References}

1 Verma S, Buchanan MR, Anderson TJ: Endothelial function testing as a biomarker of vascular disease. Circulation 2003; 108: 2054-2059.

-2 Widlansky ME, Gokce N, Keaney JF Jr, Vita JA: The clinical implications of endothelial dysfunction. J Am Coll Cardiol 2003;42: 1149-1160.

-3 Joyner MJ, Dietz NM: Nitric oxide and vasodilation in human limbs. J Appl Physiol 1997; 83:1785-1796.
4 Stocker R, Keaney JF Jr: Role of oxidative modifications in atherosclerosis. Physiol Rev 2004;84:1381-1478.

5 Feng L, Stern DM, Pile-Spellman J: Human endothelium: endovascular biopsy and molecular analysis. Radiology 1999;212:655664.
-6 Colombo PC, Ashton AW, Celaj S, Talreja A, Banchs JE, Dubois NB, Marinaccio M, Malla S, Lachmann J, Ware JA, Le Jemtel TH: Biopsy coupled to quantitative immunofluorescence: a new method to study the human vascular endothelium. J Appl Physiol 2002; 92:1331-1338.

7 Aird WC: Mechanisms of endothelial cell heterogeneity in health and disease. Circ Res 2006;98:159-162. 
$>8$ Gibbons RJ, Balady GJ, Beasley JW, Bricker JT, Duvernoy WF, Froelicher VF, Mark DB, Marwick TH, McCallister BD, Thompson PD Jr, Winters WL, Yanowitz FG, Ritchie JL, Gibbons RJ, Cheitlin MD, Eagle KA, Gardner TJ, Garson A Jr, Lewis RP, O’Rourke RA, Ryan TJ: ACC/AHA Guidelines for Exercise Testing. A report of the American College of Cardiology/American Heart Association Task Force on Practice Guidelines (Committee on Exercise Testing). J Am Coll Cardiol 1997;30:260-311.
9 Donato AJ, Eskurza I, Silver AE, Levy AS, Pierce GL, Gates PE, Seals DR: Direct evidence of endothelial oxidative stress with aging in humans: relation to impaired endothelium-dependent dilation and upregulation of nuclear factor- $\kappa$ B. Circ Res 2007;100: 1659-1666.

10 Silver AE, Beske SD, Christou DD, Donato AJ, Moreau KL, Eskurza I, Gates PE, Seals DR: Overweight and obese humans demonstrate increased vascular endothelial NAD(P)H oxidase-p47(phox) expression and evidence of endothelial oxidative stress. Circulation 2007;115:627-637.

-11 Orshal JM, Khalil RA: Gender, sex hormones, and vascular tone. Am J Physiol 2004;286:R233-R249.

$>12$ Jacoby DS, Rader DJ: Renin-angiotensin system and atherothrombotic disease: from genes to treatment. Arch Int Med 2003;163: 1155-1164.
3 Dart AM, Chin-Dusting JP: Lipids and the endothelium. Cardiovasc Res 1999;43:308322.

14 Kofler S, Nickel T, Weis M: Role of cytokines in cardiovascular diseases: a focus on endothelial responses to inflammation. Clin Sci (Lond) 2005;108:205-213.

15 Guzik TJ, Sadowski J, Kapelak B, Jopek A, Rudzinski P, Pillai R, Korbut R, Channon KM: Systemic regulation of vascular $\mathrm{NAD}(\mathrm{P}) \mathrm{H}$ oxidase activity and nox isoform expression in human arteries and veins. Arterioscler Thromb Vasc Biol 2004;24:16141620 . 